\title{
Zervix-Scheiden-Abstrich bei Teenagern
}

\begin{tabular}{|l|l|l|}
\hline R.H. & & Kaufman \\
\hline H.J. & & Spjut \\
\hline S. & Shirley & Carrig \\
\hline
\end{tabular}

Department of Obstetrics and Gynecology and Department of Pathology, Baylor University College of Medicine, Houston, Tex.

Vielc Gynäkologen und Zytologen setzen eine bestimmte Altersgrenze fest, unter welcher es ihrer Ansicht nicht notwendig ist, routinemäßige zytodiagnostische Untersuchungen zur Erfassung von Zervixkarzinomen durchzuführen. Es sind jedoch eine Reihe von Publikationen bekannt, nach welchen auch bei jugend-lichen Patienten unter 20 Jahren dysplastische und karzinomatöse Epithelveränderungen an der Portio vorkommen.

In der Zeit von Januar 1962 bis April 1964 wurden in den dem Baylor University College of Medicine angeschlossenen Kranken-häusern 1199 Zervixabstriche bei Patientinnen zwischen 13 und 19 Jahren durchgeführt. Davon waren 855 geburtshilfliche Fälle, 307 gynäkologische Fälle, 77 waren nicht näher klassifiziert. Die Beurteilung wurde in drei Kategorien - negativ, zweifelhaft und positiv - durchgeführt.

Die Auswertung ergab 1167 negative, 30 zweifelhafte und 2 positive Fälle. Beide positiven Fälle wiesen ein durch Konisation verifiziertes Carcinoma in situ auf, eines war ein geburtshilflicher, eines ein gynäkologischer Fall. Von den 30 zweifelhaften Abstrichen erwiesen sich 18 bei Wiederholungsabstrichen zweimal negativ, 2 zeigten histologisch eine geringe

Plattenepitheldysplasie, bei 3 konnten durch Konisation starke dysplastische

Epithelveränderungen nachgewiesen werden. 7 Fälle konnten nicht weiter verfolgt werden.

Die jüngste Patientin mit Carcinoma in situ war 17 Jahre alt. Bei Mädchen unter 17 Jahren fanden sich nur geringe Dysplasien.

Diese Ergebnisse bestätigen neuerlich, daß es notwendig ist, bei alien geburtshilflichen und gynäkologischen Patientinnen routinemäßige Zervixabstriche ohne Rücksicht auf eine Altersgrenze durchzuführen. Besonders notwendig scheint dies bei Frauen, die

* Originaltitel: Cervico-vaginal cytology in the teenage patient.

Kaufman, Spjut und C $\lambda$ rrig, Zervix-Scheiden-Abstrich bei Teenagern 91 keine Virgines sind oder bereits geboren haben. Derzeit ist eineUntersuchung im Gange, ob Patienten mit dysplastischen Epithel-veränderungen imjugendalter später häufiger karzinomatöse Ver-änderungen bekommen. Nach Ansicht verschiedener Autoren istdie schlechte Prognose des Karzinoms im jugendlichen Alter nichtnur eine Folge einer höheren Virulenz des Tumorgewebes, sondernauch die Folge einer verspäteten Erkennung und damit auch einesverspäteten Beginns der Behandlung. H. Holzner Aus: Acta cytol. 9: 307-313 (1965); cit. Gynäk. Rdsch. 4: 91-92 (1967) Abstrich aus Scheidenspühmg in der Hormonzytologie* Maria D. Moraes-Ruehsen und T. Masukawa 
The Johns Hopkins University and Hospital, Department of Gynecology and Obstetrics and Pathology, Baltimore, Md.

In der vorliegenden Arbeit wird die vom Patienten selbst durchgeführte Irrigationsmethode (Davis) in ihrer Verwertbarkeit mit den herkömmlichen Methoden der Abstrichentnahme durch den Arzt selbst verglichen.

Vier amenorrhoische Patientinnen wurden nach vorheriger HCG-Verabreichung mit humanem hypophysärem Gonadotropin behandelt und die Lutealphase durch Progesteronverabreichung komplettiert. Bei drei der vier Patienten konnten damit ein bis zwei ovulatorische Zyklen erreicht werden. Diese wurden durch Messung der Basaltemperatur Endometriumbiopsie

Östrogen-bestimmung im Harn und tägliche Vaginalsmears kontrolliert. Die Abstriche wurden sowohl durch den Patienten selbst mittels der Irrigationsmethode gewonnen als auch zum Vergleich durch den Arzt direkt von der Vaginalwand abgenommen. Die Auswertung erfolgte durch Bestimmung des Reifungsindex (Oberflächenzell-

* Originaltitel: The irrigation smear: Use in hormonal cytology. 